\title{
Correction to: Is there a role for ECMO-facilitated resuscitation for the management of out-of-hospital cardiac arrest (OHCA) with refractory ventricular fibrillation (VF)?
}

\author{
Lindsay Cheskes $^{1}$ (D) Sheldon Cheskes ${ }^{3,4} \cdot$ Shelley McLeod ${ }^{2,3}$
}

Published online: 19 May 2021

(c) Crown 2021

\section{Correction to: Canadian Journal of Emergency Medicine https://doi.org/10.1007/s43678-021-00123-w}

The original version of this article unfortunately contained a mistake. The sequence of the author names was incorrect. The corrected sequence is given above. The original article has been corrected.

The original article can be found online at https://doi.org/10.1007/ s43678-021-00123-w.

Lindsay Cheskes

lcheskes@toh.ca

1 Department of Emergency Medicine, The Ottawa

Hospital, University of Ottawa, 1053 Carling Ave, Ottawa,

ON K1Y4E9, Canada

2 Schwartz/Reisman Emergency Medicine Institute, Sinai Health, Toronto, ON, Canada

3 Division of Emergency Medicine, Department of Family and Community Medicine, University of Toronto, Toronto, ON, Canada

4 Sunnybrook Centre for Prehospital Medicine, Li Ka Shing Knowledge Institute, St. Michaels Hospital, Toronto, ON, Canada 